\title{
VARIABILITY OBSERVATIONS OF A COMPLETE SAMPLE OF FLAT-SPECTRUM RADIO SOURCES: PRELIMINARY RESULTS
}

\author{
A.G. GORSHKOV and V.K. KONNIKOVA \\ Sternberg Astronomical Institute, Moscow State University, Russia; E-mail: algor@sai.msu.ru \\ M.G. MINGALIEV \\ Special Astrophysical Observatory, Russian Academy of Sciences, Nizhnij Arkhyz, Russia \\ E-mail:marat@sao.ru
}

\begin{abstract}
This report presents preliminary results of daily observations, over 60 and 100 days, of a complete, flux-limited sample of radio sources with flat spectra. The existence of flicker up to $21.7 \mathrm{GHz}$ was confirmed, for sources with flat spectra, on a time-scale of 4 days. A model explaining the flux density variations of the unique radio source $0524+034$, on long and short time-scales, by an intrinsic mechanism is proposed.
\end{abstract}

Two series of daily observations of a complete, flux-limited sample of radio sources with flat spectra were carried out in January-February 1998 and May-August 1999 , with the RATAN-600 radio telescope, as part of a variability monitoring program. The observations were carried out simultaneously at the frequencies 21.7 , 11.1, 7.7, 2.3, 3.9 and $0.97 \mathrm{GHz}$. The sample was selected from the Zelenchuk $3.9 \mathrm{GHz}$ Survey (Gorshkov and Konnikova, 1995) and contains all sources with fluxes $S>200 \mathrm{mJy}$ with declinations $4^{\circ}-6^{\circ}(\mathrm{B} 1950),|b|>10^{\circ}$. During both series we observed 55 sources with flat spectra, and 31 sources with steep spectra as reference sources in our variability study.

Structure functions were used to search for variability and to define its characteristic time-scale: $D^{1}(\tau)=\left\langle[f(\tau)-f(t+\tau)]^{2}\right\rangle$, where $\tau$ is the temporal lag. The main outcome of this study is the confirmation of Heeschen's results at $9 \mathrm{~cm}$ wavelength (Heeschen, 1984; Heeschen et al., 1987): flicker of sources with with flat spectra on a time-scale of 4 days. We found evidence for this effect at 5 frequencies, after averaging the structure functions of individual sources with flat spectra.

Figure I and Table I present these results. Table I contains the mean flux density $\langle S\rangle$; the $r m s$ of the variations $r m s_{\text {var }}=r m s_{s s s}\left[2 /\left(D^{1}(1)-1\right)\right]^{0.5}$, here $r m s_{s s s}-r m s$ of the steep spectrum sources and $D^{1}(1)$ - the normalized mean structure function of the flat spectrum sources (see Figure $1 \mathrm{~b}$ ); mean index of modulation, $m=$ $\left(r m s_{\text {var }} /\langle S\rangle\right) \times 100 \%$, of sources with flat spectra at 5 frequencies. For comparison, in the two last lines are adduced $\langle S\rangle$ and indexes of modulation for sources with steep spectra.

The mean modulation index is $m \approx 1.7 \%$ at all frequencies except $21.7 \mathrm{GHz}$, where $m \approx 4.5 \%$. Thirteen objects with high individual variability were excluded 


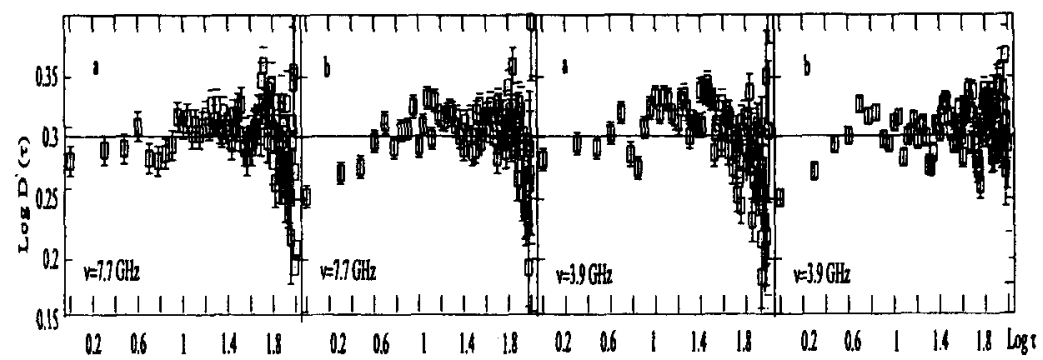

Figure 1. The normalized mean structure functions of the flux densities of sources with steep (a) and flat (b) spectra at the frequencies 7.7 and $3.9 \mathrm{GHz}$.

TABLE I

The parameters of fast variability of the sample sources

\begin{tabular}{lccccc}
\hline Frequency, GHz & 21.7 & 11.1 & 7.7 & 3.9 & 2.3 \\
\hline$\langle S\rangle$ (mJy) & 390 & 392 & 396 & 413 & 397 \\
$r m s$ of variations (mJy) & 17 & 8 & 7 & 8.5 & 7 \\
Index of modulation, $m(\%)$ & 4.5 & 2.0 & 1.7 & 1.5 & 1.7 \\
& & & & & \\
$\langle S\rangle$ (mJy) & 189 & 227 & 288 & 460 & 663 \\
Index of modulation, $m(\%)$ & 1.4 & 0.6 & 0.5 & 0.3 & 0.6 \\
\hline
\end{tabular}

from these averages. For an intrinsic origin of the variability, $T_{b}$ at $2.3 \mathrm{GHz}$ is in the range from $2.5 \cdot 10^{16}$ to $4 \cdot 10^{18} \mathrm{~K}$ for source redshifts $0.2 \leq z \leq 2$ (we employ a Hubble constant of $H_{0}=50 \mathrm{~km} \mathrm{~s}^{-1} \mathrm{Mpc}^{-1}$, and $q_{0}=0.5$ ).

Evidence was also found for variability with time-scales of 2 to 10 days, and $m=1.5-10 \%$, for 13 strong sources in the sample. This fact supports the above mentioned result. Moreover, periodic components with time-scales from 6 to 60 days were discovered in several of these objects. Sometimes the time-scale increases with increasing wavelength as, for example, in the case of $2121+053$ (Figure 2). Both kinds of variations show strong correlation over a broad frequency range.

We consider that the cause of flicker is interstellar scintillation. In the model of Rickett (1995) it is impossible to explain our data (to get observed values of $m$ in a broad frequency range). It is possible that this difficulty could be overcome by consideration of more complex source geometries. Models might be considered in which a considerable proportion of the radiation arises from structures less than a milli-arcsecond in size immersed in more extended structure. In this case there should be 1) relation between the observation frequency and the flux density 


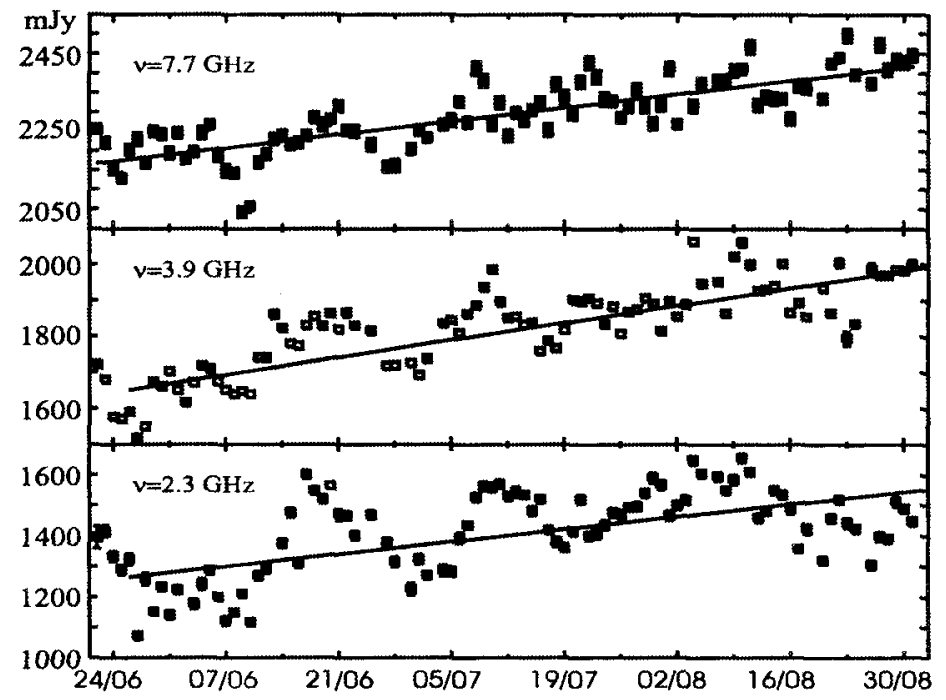

Figure 2. Light curves of the source $2121+053$ at frequencies $7.7,3.9$ and $2.3 \mathrm{GHz}$. An increase in the time-scale of variations with decreasing frequency can be seen. The periodic variations are accompanied by a long-term increase of flux density (solid line).
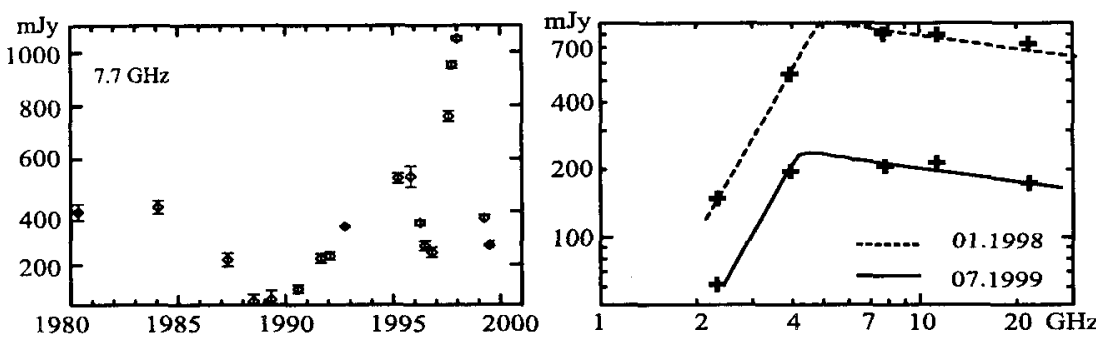

Figure 3. Long-term variations in flux density of the radio source $0524+034$ at frequency $7.7 \mathrm{GHz}$ (left), and spectra of the variable component on short time-scales at the beginning of 1998 and middle of 1999 (right).

ratio of compact and extended structures, and/or 2) dependence of angular size of compact detail from the frequency.

Long-lived periodic variations could be caused by both internal and external effects. The frequency dependence of these variations differs amongst our sources and additional observations are required to better characterize this behaviour.

Study of the variability of the unique radio source $0524+034$, on various timescales, was undertaken over a prolonged period. Long-term monitoring was begun in 1980 on the RATAN-600 telescope, and during these observations at least two flares, with time-scales of 4 and 1.5 years were discovered. The long-term variability is correlated between frequencies $21.7-2.3 \mathrm{GHz}$. The light-curve of $0524+034$ at a frequency of $7.7 \mathrm{GHz}$ is presented in Figure 3 . 
Near the maximum of the second flare, at the beginning of 1998 , variability on time-scales 6-14 days, at frequencies $21.7-2.3 \mathrm{GHz}$, was observed. The variations are correlated at all frequencies where the parameters of the variability could be determined, including the optically thick part of the spectrum. We suggested (Gorshkov et al., 2000) that the variability is due to illumination of inhomogeneities in the jet by a stock front passing through them, and that the light-curve reflects the distribution and characteristic sizes of these inhomogeneities $(0.14-0.5 \mathrm{pc}$ for angles to the line of sight not exceeding $10^{\circ}$, a Lorentz factor of 10 and an adopted redshift of $z=0.5$ ).

The simultaneity of the variations over a broad frequency range and the fact that the mean spectrum of the variable component coincides with the spectrum of a homogeneous, spherically symmetric source (Figure 3, right panel), suggest that the radiation reflects the process of acceleration of electrons and amplification of the magnetic field, with adiabatic expansion being negligible.

For the period from February 1998 through to September 1999, the mean flux density of the radio source has dropped by a factor of 5 , at the same time the relative amplitude and character of the variations on short time-scales has not changed. We suggest that the long-term variability might be explained by precession of a relativistic jet. The time-scale of variability is determined as: $t=$ $L(1-\beta \cos \theta)(1+z) / c \beta$, where $L$ is the linear dimension of the emission region, and $c \beta$ is the speed of the shock (Qian et al., 1991). In this interpretation, the decreasing flux density is connected with a decrease in the so-called Doppler factor $\Delta: S_{o b s}=S \Delta^{3+\alpha} ;$ a factor of five decline in flux then implies a decrease in $\Delta$ by a factor of 1.7. This corresponds, for our adopted parameters, to a change of the jet orientation angle by approximately $2^{\circ}$.

This work was supported by the Russian Foundation for Basic Research (9802-16428) and by the grant of the 'Universities of Russia' (5561).

\section{References}

Gorshkov, A.G. and Konnikova, V.K.: 1995, Astron. Rep. 39, 257.

Gorshkov, A.G., Konnikova, V.K. and Mingaliev, M.G.: 2000, Astron. Rep. 44, 161.

Heeschen, D.S.: 1984, Astron. J. 89, 1111.

Heeschen, D.S., Krichbaum, Th., Schalinski, C.J. and Witzel, A.: 1987, Astron. J. 94, 1493.

Qian, S.J., Quirrenbach, A., Witzel, A., Krichbaum, T.P., Hummel, C.A. and Zensus, J.A.: 1991, Astron. Astrophys. 241, 15.

Rickett, B.J., Quirrenbach, A., Wegner, R., Krichbaum, T.P. and Witzel, A.: 1995, Astron. Astrophys. 293,479 . 